\title{
Tromboembolismo pulmonar como complicación de la cetoacidosis diabética en una Unidad de Cuidado Intensivo Pediátrico: A propósito de un caso
}

\author{
Pulmonary thromboembolism as a complication of diabetic ketoacidosis \\ in a paediatric intensive care unit: case report
}

Milton Molano Trujillo', Dolly Vanessa Rojas M. ${ }^{2}$, Camilo Ernesto Ospino B. ${ }^{2}$

\begin{abstract}
Resumen
La enfermedad trombótica en el paciente pediátrico críticamente enfermo se ha convertido progresivamente en una importante causa de morbimortalidad, atribuido a la mejoría en la supervivencia de enfermedades previamente letales, el aumento del uso de catéteres venosos centrales y la creciente disponibilidad de métodos no invasivos para su diagnóstico. Se pretende con este artículo presentar un caso de TEP en un lactante mayor, en el curso de una cetoacidosis diabética con un factor de riesgo claro debido al uso de un dispositivo vascular. En estos pacientes es alta la probabilidad de desarrollar complicaciones tromboembólicas y por tal razón se considera racional el uso de regímenes de profilaxis antitrombótica.
\end{abstract}

Palabras clave: pediatría, tromboembolismo pulmonar, cetoacidosis diabética, cuidado intensivo pediátrico, catéteres vasculares.

\begin{abstract}
Pulmonary thromboembolism in a critically ill paediatric patient has steadily become a significant cause of morbidity and mortality, attributed to the improvement in survival rates from previously fatal diseases, the increase in the use of central venous catheters and growing availability of non-invasive diagnostic methods. This article intends to present a case of pulmonary thromboembolism in a breastfed baby suffering from diabetic ketoacidosis with a clear risk factor due to the use of a vascular device. In these patients there is a high probability of developing thromboembolic complications and therefore the use of antithrombotic prophylactic regimens is deemed reasonable.
\end{abstract}

Key words: Paediatrics, Pulmonary Thromboembolism, Diabetic Ketoacidosis, Paediatric Intensive Care Unit, Vascular Catheters

1. MD. Pediatra, Docente Universidad Surcolombiana, Unidad de Cuidados Intensivos Pediátrico. Hospital Universitario de Neiva, Colombia.

2. MD. Pediatra, Universidad Surcolombiana, Hospital Universitario de Neiva, Colombia.

Correspondencia: Dolly Vanessa. Correo electrónico: dollyvanessa@gmail.com

Recibido: 14/03/2015-Revisado: 29/03/2015-Aceptado: 13/10/2015 
R.F.S Revista Facultad de Salud

Julio-Diciembre de 2015;7(2):51-56
Tromboembolismo pulmonar como complicación de la cetoacidosis diabética en una Unidad de Cuidado Intensivo Pediátrico: A propósito de un caso

\section{Introducción}

La enfermedad trombótica (ET) en el paciente pediátrico críticamente enfermo se ha convertido progresivamente en una importante causa de morbilidad y mortalidad en esta población vulnerable. Los factores de riesgo más importantes en pediatría incluyen catéteres vasculares, enfermedad cardiaca congénita y malignidad; escenarios comunes en las Unidades de Cuidado Intensivo Pediátrico (UCIP) ${ }^{1}$. Sin embargo, en pacientes con Cetoacidosis Diabética (CAD) tal riesgo parece estar incrementado. Dentro del espectro de la ET se encuentra el tromboembolismo pulmonar (TEP) evento poco común en niños, pero que en las últimas dos décadas ha aumentado su diagnóstico atribuido a la mejoría en la supervivencia de enfermedades de la infancia previamente letales, el aumento del uso de catéteres venosos centrales y el incremento de la disponibilidad de métodos no invasivos para su diagnóstico ${ }^{2}$. Los signos clínicos no son específicos en niños y pueden ser enmascarados por las condiciones subyacentes. Se pretende con este artículo presentar un caso de TEP en un lactante mayor en quien se documentó el desarrollo de la patología, durante el curso de una cetoacidosis como debut de su diabetes mellitus.

\section{Presentación del caso}

Paciente de 2 años, sexo masculino, sin antecedentes relevantes. Presenta cuadro de 15 días de evolución consistente en astenia, adinamia y pérdida de peso subjetiva. Cinco días antes de la primera consulta inicia cuadro respiratorio alto manejado con antibiótico oral sin mejoría. Progresa a dificultad respiratoria y alteración de la conciencia. Es llevado para atención médica en primer nivel, durante la valoración toman glucometría: "HIGH" y entonces remiten a hospital de II nivel donde se hace diagnóstico de diabetes tipo I de novo y CAD, administran infusión de insulina, reposición de líquidos intravenosos y remiten a UCIP en III nivel de atención. Al ingreso a la UCIP se encuentra deshidratada, grado II, en el curso de una CAD severa, acidosis metabólica severa con anión GAP elevado y compromiso del estado de conciencia. Fue necesario asegurar vía aérea y ventilación mecánica. El acceso venoso de logra con catéter venoso central femoral derecho, sin complicaciones. Se ajusta infusión de líquidos endovenosos, infusión de insulina además de cubrimiento antibiótico por la sospecha de cursar con cuadro séptico. Se toman tomografía cerebral simple y placa de Rx de tórax reportados como normales. Presenta evolución hacia la mejoría, lográndose pasar a insulinas análogas. El 2 día hospitalario surge la necesidad de aumentar la fracción inspirada de oxígeno, SAFI: 188 y edema en miembro inferior derecho. Se describe rectorragia escasa, disminución del nivel de hemoglobina y trombocitopenia. Se ordena radiografía de tórax portátil de control (Figura 1) y ecografía doppler venoso de miembros inferiores el cual se reportó con trombosis venosa femoral derecha.

Paraclínicos muestran dímero D: $1752 \mathrm{ng} / \mathrm{mL}$. Tiempos de coagulación TP normal, TTP 44.9seg., control $29.1 \mathrm{seg}$.

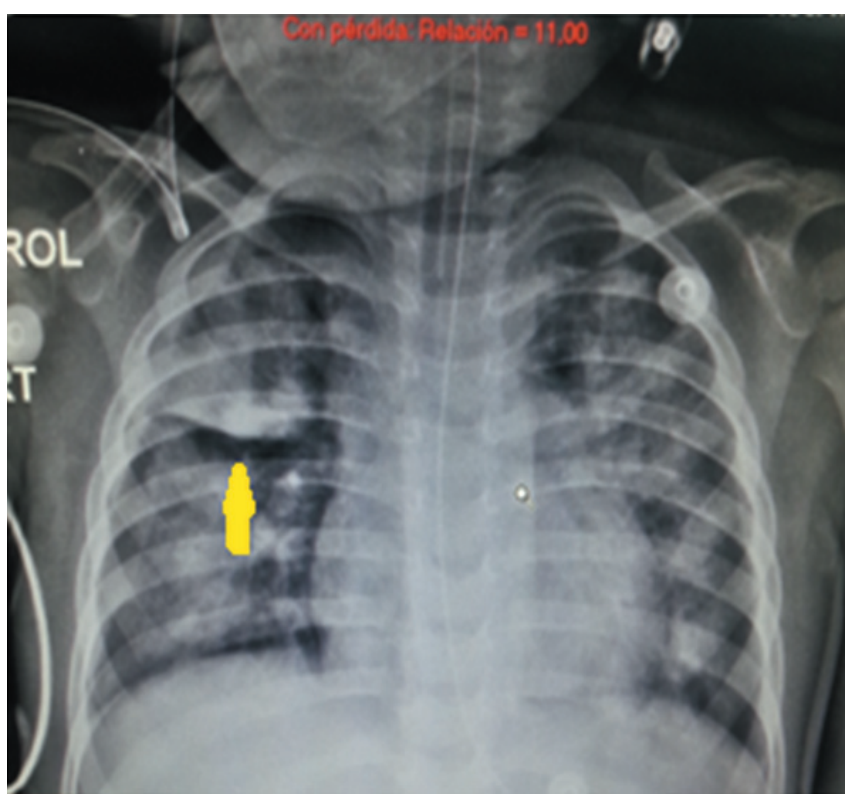

Figura 1. Rx. portátil de tórax control 3 día hospitalario. Imagen sugestiva de infiltrados de ocupación alveolar apicales derechos, y alternancia de áreas radiolúcidas y áreas de opacidad alveolar. (Bandas de Mach).

INR 1.07. Control a las 24 horas: dímero D $5287 \mathrm{ng} / \mathrm{mL}$. y TTP que no coagula. Ecografía doppler de vasos renales normal, Angiotac de tórax donde se observan fallas endoluminales en la arteria pulmonar derecha, así como en las segmentarias bilateralmente. Hay obliteración en la arteria pulmonar derecha del 20\% sugestivo de trombos endoluminales. (Figura 2) Ecocardiograma de enero 19 de 2013 con disfunción miocárdica biventricular fracción de eyección del 40\% Fracción de acortamiento de $19 \%$, insuficiencia mitral grado I, PSPE de $33 \mathrm{mmHg}$. Se inicia anticoagulación plena con heparina no fraccionada y dos días después se pasa a heparina de bajo peso molecular. Evolución hacia la mejoría del estado hemodinámico, ventilatorio y metabólico además mejoría radiológica y hematológica. Control ecocardiográfico de noviembre 21 de 2013 que reporta fracción de eyección de 59\%, fracción de acortamiento de $31 \%$, corazón estructuralmente normal, con buena función sisto-diastólica del ventrículo izquierdo. Estancia en UCI pediátrica 15 días. Sale a hospitalización en piso de pediatría general, recibiendo heparina de bajo peso molecular SC.

\section{Discusión}

La ET en el niño críticamente enfermo se ha convertido en una importante causa de morbimortalidad. La prevalencia actual es de 0,5 a 0,14/10000 niños. En niños el 80-90\% tienen uno o más factores de riesgo subyacentes, afecta de forma similar el sistema venoso superior como en el inferior, mientras que en adultos $95 \%$ se presentan en éste último. La más alta prevalencia se ha visto en menores de 2 años, como nuestro paciente; con un segundo pico en la adolescencia. La ET 

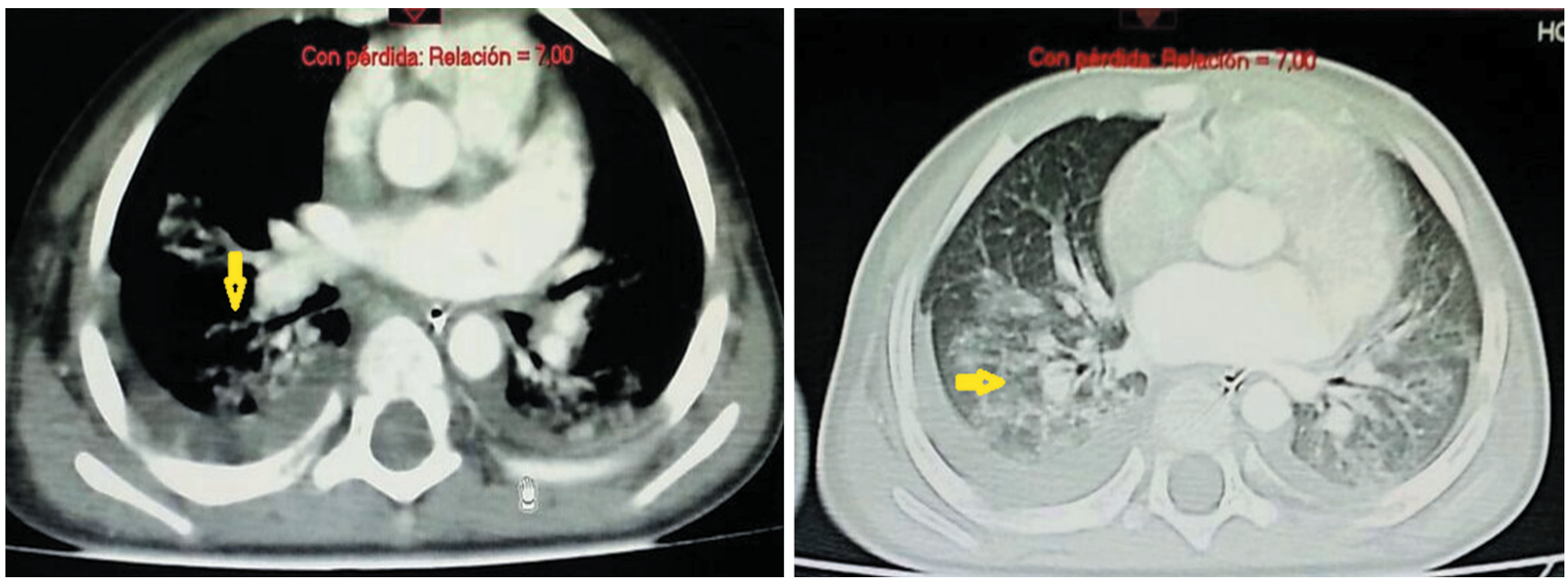

Figura 2. Angio TAC de tórax simple y contrastado.

ocurre en igual proporción hombre: mujer en <11años, sin embargo en adolescentes predomina en mujeres 2:1 atribuible a factores como el embarazo y el uso de anticonceptivos. En el contexto del paciente pediátrico ortopédico, el estudio POSNA, reporta como principales factores de riesgo el antecedente de un evento trombótico y la presencia de $\mathrm{CVC}^{3}$.

La fisiopatología de la ET en pacientes con CAD no está claramente entendida. Desde 1845, Virchow planteó la interacción de tres elementos: hipercoagulabilidad, estasis del flujo sanguíneo y daño endotelial para el desarrollo de trombosis ${ }^{1}$.

Frente a lo primero, es reconocido que la diabetes, per se, condiciona a un estado hipercoagulable, marcadores de activación de la coagulación, tales como los fragmentos de activación de protrombina 1 y 2 , a la par los complejos trombina - antitrombina, se encuentran elevados en esta patología, al igual que el fibrinógeno, factores VII, VIII, XI y XII, calicreína y el factor de von Willebrand. Por el contrario, el nivel de la proteína $\mathrm{C}$ se reduce y el sistema fibrinolítico, se encuentra relativamente inhibido ${ }^{4}$.

En cuanto a la estasis sanguínea, la hiperglicemia al incrementar marcadamente la agregación plaquetaria y la rigidez de la membrana de los glóbulos rojos ocasiona resistencia al flujo, mediante diversos mecanismos como la disminución en la disponibilidad del óxido nítrico ${ }^{5}$.

$Y$ finalmente haciendo referencia al daño endotelial, los catéteres vasculares se constituyen en el factor de riesgo más comúnmente identificado en lactantes y niños. Independientemente del sitio de inserción, son trombogénicos, constituyéndose en cuerpos extraños con superficies no endoteliales que actúan como nido para la activación de la cascada de coagulación ${ }^{1}$.

Gutiérrez, J. et al, reportan un estudio retrospectivo de casos y controles, realizado en pacientes admitidos por CAD a las UCIP quienes requirieron colocación de CVC femoral. La mitad de los pacientes con CAD desarrollaron TVP, mientras que ninguno de los controles desarrolló el fenómeno trombótico $(\mathrm{p}<0.007)$, considerando racional el uso de regímenes de profilaxis antitrombótica en esta población ${ }^{5}$.

Las manifestaciones clínicas de la ET en la población pediátrica dependen del espectro clínico. Los síntomas comúnmente reportados incluyen dolor, edema, pérdida de la permeabilidad o función de $\mathrm{CVC}$, hipoxia, dificultad respiratoria y disfunción neurológica ${ }^{1}$. En adultos se han validado escalas de predicción clínica como el puntaje de Well y el de Geneva, que combinan signos clínicos y factores de riesgo para evaluar su probabilidad. En niños no han sido validadas, por tanto, es importante explorar la presencia de los factores de riesgo, que generen la sospecha e iniciar la evaluación diagnóstica de la enfermedad ${ }^{2}$.

Debido a la falta de especificidad de los signos clínicos de la ET, el diagnóstico por imágenes y por laboratorio se hace necesario. El dímero D resulta elevado en eventos trombóticos ${ }^{2}$, con alto VPN pero con la desventaja de presentar falsos positivos en condiciones tales como la misma diabetes, el embarazo, desórdenes gastrointestinales, entre otros ${ }^{6}$.

Aunque la venografía contrastada fue el estándar de oro para el diagnóstico de TVP, por mucho tiempo, su carácter invasivo y exposición a radiación, han hecho que sea reemplazada de manera paulatina por el Ultrasonido Doppler (US), no invasivo, menos costoso, y con mejor sensibilidad $97 \%{ }^{6}$. Sin embargo no siempre está disponible inmediatamente. Tay ET, et al, en el 2012 hacen las descripción de 3 casos de TVP en adolescentes identificados mediante ecografía de emergencia en la sala de urgencias pediátricas. Este reporte de casos resalta la importancia del US de emergencias realizado por personal no radiólogo, como herramienta útil para la identificación temprana de esta patología ${ }^{7}$. Con base a los factores de riesgo, los estudios de laboratorio e imagenológicas iniciales se han creado diversos algoritmos, para el diagnóstico de TVP y TEP (Figuras 3 y 4).

La venografía por tomografía computarizada espiral es el estudio diagnóstico de elección para los niños con sospecha de TEP. La tomografía de Ventilación/perfusión resulta 
R.F.S Revista Facultad de Salud

Julio-Diciembre de 2015;7(2):51-56
Tromboembolismo pulmonar como complicación de la cetoacidosis diabética en una Unidad de Cuidado Intensivo Pediátrico: A propósito de un caso

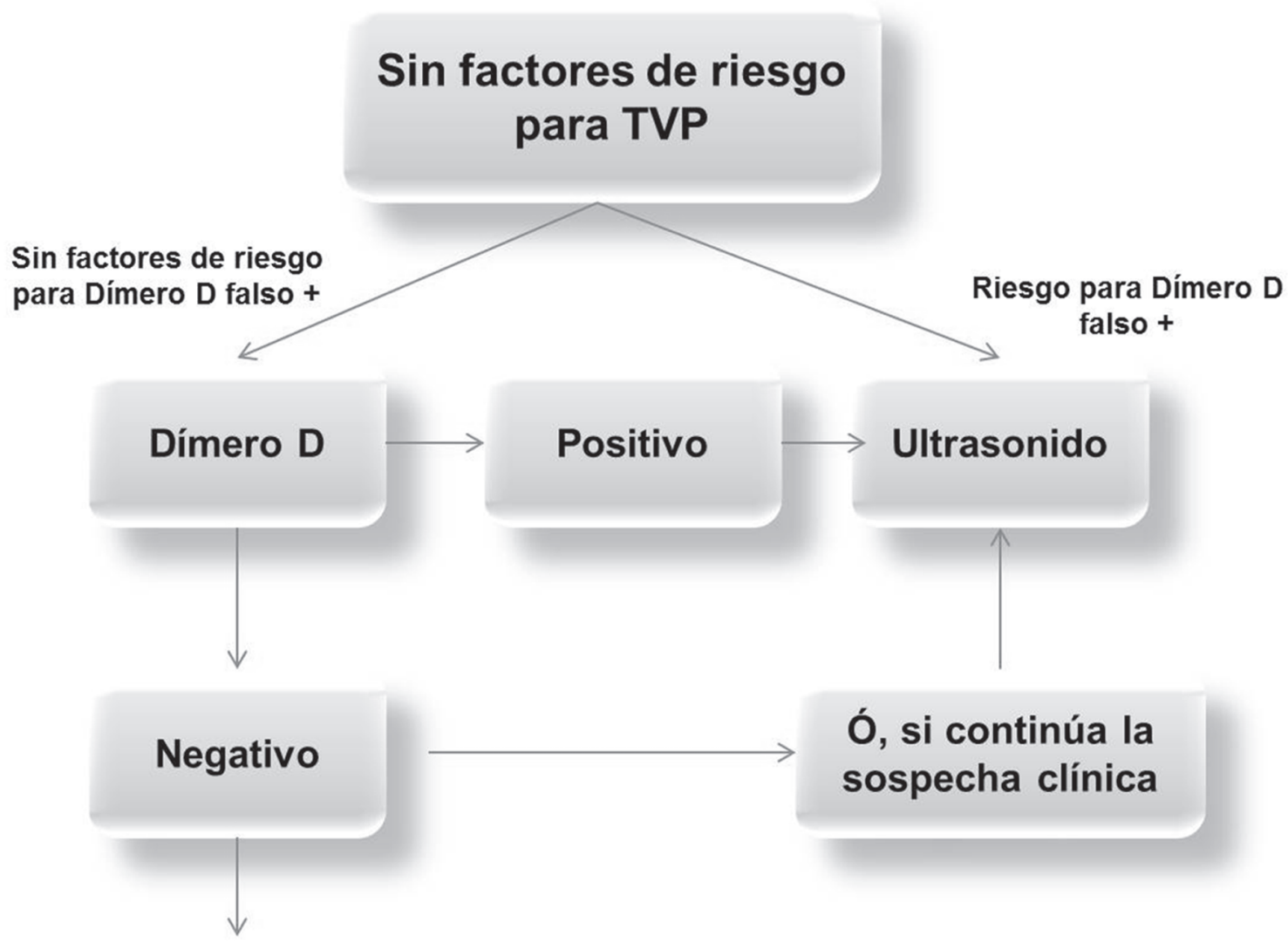

ALTO

Figura 3. Algoritmo paciente con factores de riesgo para TVP.

menos útil por la dificultad en su interpretación en pacientes críticamente enfermos con otros desórdenes cardíacos o respiratorios ${ }^{1}$.

El tratamiento de la ET en la población pediátrica involucra la corrección de los factores de riesgo fácilmente modificables, tales como remover catéteres vasculares. La medicación antitrombótica para niños críticamente enfermos incluyen heparina no fraccionada (HNF), heparina de bajo peso molecular (HBPM), inhibidores directos de la trombina $\mathrm{y}$ activadores del plasminógeno ( $\mathrm{tPa}$, urokinasa, estreptoquinasa), mientras que el manejo crónico de la trombosis incluye antagonistas de la vitamina $\mathrm{K}$ o $\mathrm{HBPM}^{1}$. La HNF ofrece varias ventajas, por su vida media corta, parámetros de laboratorio bien estudiados y un antídoto disponible (protamina). La dosis de carga recomendada es 75$100 \mathrm{U} / \mathrm{kg}$ IV y mantenimiento de $20-28 \mathrm{U} / \mathrm{kg} / \mathrm{h}$ para niños y lactantes respectivamente. La enoxaparina es la HBPM más utilizada, las dosis para lactantes son de $1,5 \mathrm{mg} / \mathrm{kg}$ cada 12 horas, y para niños $1 \mathrm{mg} / \mathrm{kg}$ cada 12 horas, con el objetivo de mantener el INR: 2-3. Para el manejo crónico de ciertas enfermedades que condicionan a la trombosis se encuentran indicados los antagonistas de la vitamina $\mathrm{K}$ como la warfarina. La dosis de carga recomendada es de $0,2 \mathrm{mg} / \mathrm{kg}$ y posterior ajuste de acuerdo a los valores del $\mathrm{INR}^{1}$.
En general, se recomienda, después de un primer episodio de trombosis idiopática, sintomática o persistente, en un paciente mayor de 2 meses, garantizar el tratamiento con heparina durante la fase aguda (5-10 días), seguidos de al menos 3-6meses de anticoagulación continuada con warfarina o HBPM subcutáneas. Posteriormente, los pacientes deben ser reevaluados para determinar el riesgo/beneficio de extender la terapia anticoagulante. El riesgo de recurrencia es mayor en paciente con TVP residual en US repetitivos, considerándose como un factor de riesgo independiente para tromboembolismo recurrente ${ }^{1}$.

Montes et al publican en el 2013 un estudio realizado en 125 pacientes, todos diagnosticados con TVP de los miembros inferiores, encontrando una correlación negativa significativa entre los niveles de dímero $\mathrm{D}$ a los 3 meses y la mejoría de la trombosis residual $(\mathrm{P}=0.016)$, por el contrario una correlación positiva significativa entre el diámetro del trombo $\mathrm{y}$ los niveles de IL-8 ( $\mathrm{P}=0.015)$. Este estudio resalta la baja frecuencia de la normalización posterior a un periodo estándar de anticoagulación y su relación con diversos biomarcadores, sugiriendo más estudios para poder ser implementados en el seguimiento de estos pacientes ${ }^{8}$.

En niños quienes tienen contraindicada la anticoagulación o tienen TEP recurrente se encuentran indi- 


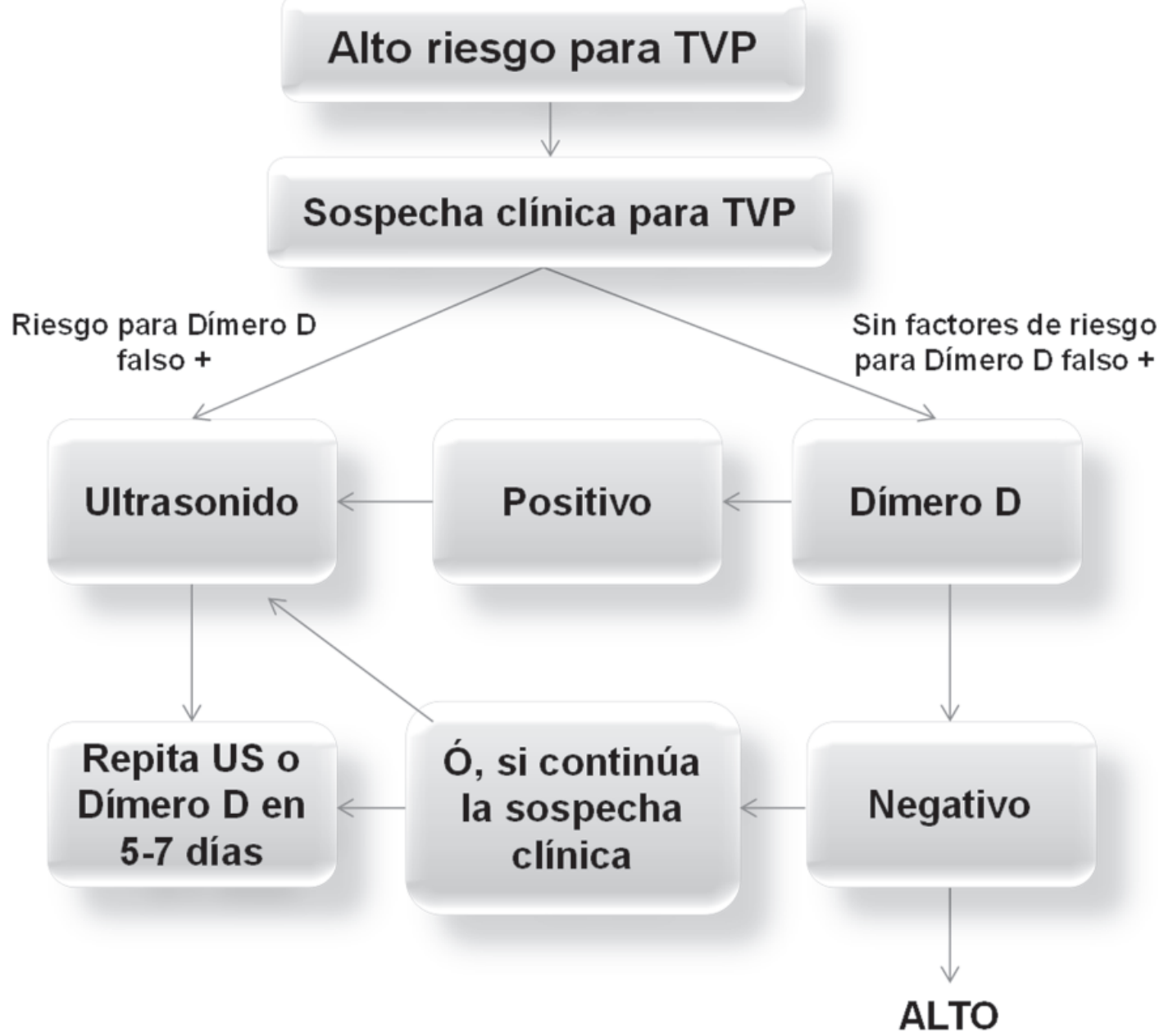

Figura 4. Algoritmo para paciente con alto riesgo de TVP.

cados los filtros de vena cava inferior. Sin embargo son muy pocos los estudios a largo plazo sobre su utilización en niños. Kukreja y colaboradores realizaron una revisión retrospectiva de 12 años en niños quienes requirieron la colocación de este tipo de dispositivo. Sus resultados indican que los filtros de VCI pueden ser colocados y retirados exitosamente en niños, con mínimas complicaciones relacionadas con el procedimiento $^{9}$.

De igual forma, en el seguimiento de los niños que han padecido de episodios de TVP, es importante tener en cuenta la posible aparición del síndrome postrombótico (SPT), caracterizado por dolor, edema, decoloración de la piel y ulceración posterior al daño valvular por el trombo lo que contribuye a insuficiencia venosa crónica. Aunque ha sido más estudiado en adultos, su reconocimiento se ha hecho más frecuente en la población pediátrica. Para ello Sharathkumar y colaboradores, realizan un estudio que evalúa el SPT en niños. En este estudio el SPT se desarrolló en $20 \%$ de los pacientes estudiados, especialmente en aquellos en quienes se retrasó el inicio del tratamiento ( $>48 \mathrm{~h})$ o recurrió la TVP $(\mathrm{P}<0,05)^{10}$.

\section{Conclusión}

La ET es cada vez más frecuente en el paciente pediátrico críticamente enfermo, En especial, en pacientes con CAD quienes tienen mayor riesgo de desarrollar complicaciones tromboembólicas debido a las características fisiopatológicas de la enfermedad. Asociado, el uso de dispositivos vasculares como los CVC, puede incrementar la probabilidad de desarrollar complicaciones trombóticas, considerándose racional el uso de regímenes de profilaxis antitrombótica en esta población.

\section{Referencias}

1. McCrory MC, Brady KM, Takemoto C, et al. Thrombotic disease in critically ill children. Pediatric Critical Care Medicine 2011;12: 80-89.

2. Dijk FN, Curtin J, Lord D, et al. Pulmonary Embolism in Children. Paediatric Respiratory Reviews 2012;13:112-122.

3. Sabharwal S, Passannante MR. Venous Thromboembolism in Children: Preliminary Results of a Survey of POSNA Members. J Pediatr Orthop 2013;33:852-856. 
R.F.S Revista Facultad de Salud

Julio-Diciembre de 2015;7(2):51-56
Tromboembolismo pulmonar como complicación de la cetoacidosis diabética en una Unidad de Cuidado Intensivo Pediátrico: A propósito de un caso
4. Carr ME. Diabetes mellitus: a hypercoagulable state. Journal of Diabetes Complications. 2001;15:44-54.

5. Gutierrez JA, Bagatell R, Samson MP, et al. Femoral central venous catheter-associated deep venous thrombosis in children with diabetic ketoacidosis. Critical Care Medicine 2003;3:80-83.

6. Andrews EJ, Fleische AC. Sonography for Deep Venous Thrombosis Current and Future Applications. Ultrasound Quarterly 2005;21:213-225.

7. Tay ET, Stone MB, Tsung James W. Emergency Ultrasound Diagnosis of Deep Venous Thrombosis in the Pediatric Emergency Department, A Case Series. Pediatric Emergency Care 2012;28:90-95.
8. Montes A, Arellano E, Elías T, León J, Rodriguez JA, Otero R. Residual thrombosis after a first episode of proximal deep venous thrombosis. Blood Coagulation and Fibrinolysis 2013;24:355-360.

9. Kukreja KU, Gollamudi J, Patel MN, et al. Inferior Vena Cava Filters in Children: Our Experience and Suggested Guidelines. Journal of Pediatric Hematology and Oncology $2011 ; 33: 334-338$.

10. Sharathkumar AA, Pipe SW. Post-thrombotic Syndrome in Children: A Single Center Experience. Journal of Pediatric Hematology and Oncology 2008;30:261-266. 